\title{
The Cohomology of the Space of Magnetic Monopoles
}

\author{
Graeme Segal ${ }^{1}$, Alex Selby ${ }^{2, \star}$ \\ ${ }^{1}$ DPMMS, 16 Mill Lane, Cambridge, CB2 1SB, England \\ ${ }^{2}$ New Hall, Cambridge, CB3 0DF, England
}

Received: 8 April 1995/Accepted: 12 June 1995

\begin{abstract}
Denote by $X_{q}$ the reduced space of $S U_{2}$ monopoles of charge $q$ in $\mathbb{R}^{3}$. In this paper the cohomology of $X_{q}$, the cohomology with compact supports of $X_{q}$, and the image of the latter in the former are all calculated as representations of $\mathbb{Z} / q \mathbb{Z}$ which acts on $X_{2}$. This provides a non-trivial "lower bound" for the $L^{2}$ cohomology of $X_{q}$ which is compatible with some conjectures of Sen. It is also shown that, granted some assumptions about the metric on $X_{q}$, its $L^{2}$ cohomology does not exceed this bound in the situation referred to in the paper as the "coprime case".
\end{abstract}

\section{Introduction}

The moduli space $\mathscr{M}_{q}$ of $S U_{2}$-monopoles of magnetic charge $q$ in $\mathbb{R}^{3}$ is a Riemannian manifold of dimension $4 q$. It has remarkable geometric properties, of which a comprehensive account can be found in $[\mathrm{A}-\mathrm{H}]$. Recently, to test hypotheses concerning electric-magnetic duality in non-abelian gauge theories [Sen], there has been interest in determining the square-summable harmonic forms on $\mathscr{M}_{q}$ - or, more precisely, on a $(4 q-4)$-dimensional "reduced" moduli space $X_{q}$ contained in it. To define the reduced space we first get rid of the free action of the group $\mathbb{R}^{3}$ of translations by restricting to monopoles whose centre of mass is at the origin in $\mathbb{R}^{3}$. There is still a free action of the circle group $\mathbb{T}$ which rotates the "phase" of a monopole. We cannot normalize the phase away completely, but we can fix it up to a $q^{\text {th }}$ root of unity. This gives us a simply connected manifold $X_{q}$, on which the cyclic group $\mu_{q}$ of $q^{\text {th }}$ roots of unity still acts freely by rotating the phase.

Let $\mathscr{H}_{q}^{i}$ denote the space of square-summable harmonic $i$-forms on $X_{q}$. We can decompose $\mathscr{H}_{q}^{i}$ according to the induced action of $\mu_{q}$

$$
\mathscr{H}_{q}^{i}=\bigoplus \mathscr{H}_{q, p}^{i},
$$

where $\mathscr{H}_{q, p}^{i}$ is the part where the elements $\zeta \in \mu_{q}$ act by multiplication by $\zeta^{p}$. Sen

\footnotetext{
$\star$ The work described here was carried out partly at the University of Texas at Austin.
} 
[Sen] has conjectured that

(i) If $p$ and $q$ are relatively prime then $\mathscr{H}_{q, p}^{i}=0$ except in the middle dimension $i=2 q-2$, in which $\mathscr{H}_{q, p}^{2 q-2} \cong \mathbb{C}$, and

(ii) if $q$ and $p$ have a common factor then $\mathscr{H}_{q, p}^{i}=0$ for all $i$.

In fact it is conjectured that there is a natural action of the group $S L_{2} \mathbb{Z}$ on the $L^{2}$ harmonic forms on all of the $X_{q}$ together, which transforms the $(p, q)$-bigrading according to the natural action of $S L_{2} \mathbb{Z}$ on $\mathbb{Z}^{2}$.

In this paper we shall provide some evidence in support of Sen's conjecture by calculating the cohomology of the space $X_{q}$ together with the action of $\mu_{q}$ on it. In particular we shall show that part (i) of the conjecture follows from some mild and very plausible assumptions about the nature of the metric on $X_{q}$.

We begin by stating our results about the cohomology. We write $H^{*}\left(X_{q}\right)$ for the cohomology with complex coefficients, and $H^{*}\left(X_{q}\right)_{p}$ for the part where the group $\mu_{q}$ acts by $\zeta \mapsto \zeta^{p}$.

\section{Theorem 1.1.}

$$
\begin{aligned}
H^{i}\left(X_{q}\right)_{p} & \cong \mathbb{C} \quad \text { if } i=2 q-2(p, q) \\
& =0 \text { otherwise. }
\end{aligned}
$$

Here $(p, q)$ denotes the greatest common divisor of $p$ and $q$.

This result is compatible with the existence of an $S L_{2} \mathbb{Z}$-action on $\bigoplus_{p, q} H^{*}\left(X_{q}\right)_{p}$ which does not preserve the dimension of the cohomology classes, but does preserve the dimension relative to the middle dimension $2 q-2$.

Because $X_{q}$ is an orientable open manifold of dimension $4 q-4$ we can deduce its cohomology with compact supports from Theorem 1.1 by Poincare duality.

\section{Corollary 1.2.}

$$
\begin{aligned}
& H_{c p t}^{i}\left(X_{q}\right)_{p} \cong \mathbb{C} \quad \text { if } i=2 q+2(p, q)-4 \\
&=0 \text { otherwise. }
\end{aligned}
$$

The map $H_{c p t}^{i} \rightarrow H^{i}$ is trivially zero except perhaps in the middle dimension $i=2 q-2$. We shall prove

\section{Theorem 1.3.}

$$
H_{c p t}^{2 q-2}\left(X_{q}\right) \stackrel{\cong}{\rightarrow} H^{2 q-2}\left(X_{q}\right) .
$$

In view of these topological results the following arguments show that the spaces $\mathscr{H}_{q, p}^{i}$ of $L^{2}$ harmonic forms are at least as large as Sen conjectures.

First, because $X_{q}$ is a complete Riemannian manifold, we know ([deR] Sect. 35 Theorem 26) that any $L^{2}$ harmonic form on it is closed and coclosed, and so there is a map

$$
\mathscr{H}_{q, p}^{i} \rightarrow H^{i}\left(X_{q}\right)_{p}
$$

We also know ([deR] Sect. 32 Theorem 24) that the space $\Omega_{\left(L^{2}\right)}$ of all $L^{2}$ forms on $X_{q}$ has an orthogonal decomposition

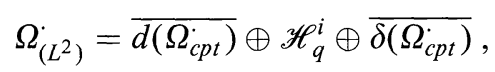


where $\Omega_{c p t}$ denotes the smooth forms with compact support, and the bars denote the closure in $L^{2}$. So there is a map

$$
H_{c p t}^{i}\left(X_{q}\right) \rightarrow \mathscr{H}_{q}^{i}
$$

defined by orthogonal projection of smooth closed forms with compact support. We have

Lemma 1.4. The composition

$$
H_{c p t}\left(X_{q}\right) \rightarrow \mathscr{H}_{q}^{\cdot} \rightarrow H^{\cdot}\left(X_{q}\right)
$$

is the obvious map.

Proof. The map takes a closed form $\alpha \in \Omega_{c p t}$ to $\alpha-\beta$, where $\beta=\lim d \gamma_{i}$ in $L^{2}$, with $\gamma_{i} \in \Omega_{c p t}$. But $\beta$ represents zero in $H^{\cdot}\left(X_{q}\right)$ because of Poincaré duality, for

$$
\int \beta \theta=\lim \int d\left(\gamma_{i} \theta\right)=0
$$

for any closed $\theta \in \Omega_{c p t}$.

Thus Theorem 1.3 tells us that $\operatorname{dim}\left(\mathscr{H}_{q, p}^{2 q-2}\right) \geqq 1$ when $p$ and $q$ are coprime. We have not used any property of the metric of $X_{q}$ except completeness. To prove that $\operatorname{dim}\left(\mathscr{H}_{q, p}^{2 q-2}\right) \leqq 1$, in the coprime case, we need some more precise properties of the metric. This is explained in Sect. 3.

\section{Calculation of the Cohomology}

The proofs of Theorems 1.1 and 1.3 depend on two elementary observations. First, the functor $X \mapsto H^{*}(X)_{p}$ is a cohomology theory on the category of spaces with $\mu_{q}$-action, in the sense that after taking the $p$-component we still have a long exact sequence associated to a space and a subspace, and we have a Mayer-Vietoris sequence for the cohomology of a union. Secondly, if $g$ is a generator of $\mu_{q}$, and $g^{k}$ is homotopic to the identity on $X$, then $H^{*}(X)_{p}=0$ unless the order of the root of unity $e^{2 \pi i p / q}$ divides $k$, i.e. unless $k p$ is divisible by $q$.

We shall use different descriptions of $X_{q}$ for the proofs of the two theorems. For Theorem 1 we use Donaldson's identification of the moduli space $\mathscr{M}_{q}$ with the space of rational functions of the form

$$
\frac{\varphi}{\psi}=\frac{a_{q-1} z^{q-1}+\cdots+a_{1} z+a_{0}}{z^{q}+b_{q-1} z^{q-1}+\cdots+b_{1} z+b_{0}},
$$

where $\varphi$ and $\psi$ are polynomials with complex coefficients and no common root. The last condition can be expressed

$$
\mathscr{R}(\varphi, \psi) \neq 0,
$$

where the resultant $\mathscr{R}(\varphi, \psi)$ is the polynomial in the $a_{i}$ and $b_{i}$ defined by

$$
\mathscr{R}(\varphi, \psi)=\prod \varphi\left(\beta_{l}\right),
$$

where $\beta_{1}, \ldots, \beta_{q}$ are the roots of $\psi$. We notice that $\mathscr{R}(\varphi, \psi)$ is homogeneous of degree $q$ in $a_{0}, \ldots, a_{q-1}$. The group $\mathbb{T}$ acts on $\mathscr{M}_{q}$ simply by multiplication. The reduced moduli space $X_{q}$ is the subspace of $\mathscr{M}_{q}$ defined by the two conditions 
$b_{q-1}=0$ and $\mathscr{R}(\varphi, \psi)=1$. It is a nonsingular algebraic hypersurface in $\mathbb{C}^{2 q-1}$. (The hypersurfaces defined by $\mathscr{R}(\varphi, \psi)=\lambda$ must be nonsingular for almost all $\lambda$; but by the homogeneity of $\mathscr{R}(\varphi, \psi)$ they are all isomorphic when $\lambda \neq 0$.)

Proof of Theorem 1.1. We first consider the part of $H^{*}\left(X_{q}\right)$ fixed under the $\mu_{q}$-action, i.e. the case $p=0$. Then $H^{*}\left(X_{q}\right)_{0} \cong H^{*}\left(X_{q} / \mu_{q}\right)=H^{*}\left(\mathscr{M}_{q} / \mathbb{C}^{\times}\right)$. The theorem asserts that $\mathscr{M}_{q} / \mathbb{C}^{\times}$has the rational homotopy type of a point. Now $\pi_{1}\left(\mathscr{M}_{q}\right)=\mathbb{Z}$, and the map $\mathscr{M}_{q} \rightarrow \mathbb{C}^{\times}$defined by $\varphi / \psi \mapsto \mathscr{R}(\varphi, \psi)$ induces an isomorphism of rational homology. (This is a simple consequence of the results of [S], where it is proved that $H_{*}\left(\mathscr{M}_{q}\right)$ - with arbitrary coefficients - is a summand in $H_{*}\left(\Omega^{2} S^{3}\right)$; for, rationally, we have $\Omega^{2} S^{3} \simeq S^{1}$.) The multiplication action of $\mathbb{C}^{\times}$on $\mathscr{M}_{q}$ gives us maps $\mathbb{C}^{\times} \rightarrow \mathscr{M}_{q}$, and the composites $\mathbb{C}^{\times} \rightarrow \mathscr{M}_{q} \rightarrow \mathbb{C}^{\times}$are $u \mapsto c u^{q}$. Homotopically, therefore, $\mathscr{M}_{q} / \mathbb{C}^{\times}$is a bundle over the classifying space $B \mathbb{C}^{\times}=\mathbb{C} P^{\infty}$ with fibre $\mathscr{M}_{q}$, and so it is rationally a circle bundle over $\mathbb{C} P^{\infty}$ with Chern class $q \in H^{2}\left(\mathbb{C} P^{\infty}, \mathbb{Z}\right)=\mathbb{Z}$. The Gysin sequence for this circle bundle shows that $\mathscr{M}_{q} / \mathbb{C}^{\times}$is rationally a point.

To treat the other cases of Theorem 1.1 we stratify $X_{q}$ according to the multiplicities of the roots of $\psi$ : if $\lambda=\left(\lambda_{1} \geqq \lambda_{2} \geqq \cdots \geqq \lambda_{r}\right)$ is a partition of $q$ we write $X_{\lambda}$ for the part of $X_{q}$ where the denominator $\psi$ is of the form

$$
\psi=\prod_{i=1}^{r}\left(z-\beta_{i}\right)^{\lambda_{l}}
$$

with $\beta_{1}, \ldots, \beta_{r}$ distinct.

If $p$ is a non-zero residue class modulo $q$, let $l>1$ divide the order of the root of unity $e^{2 \pi i p / q}$. Let $Y$ be the part of $X_{q}$ consisting of rational functions $\varphi / \psi$ such that $\psi=\chi^{l}$ for some polynomial $\chi$ of degree $r=q / l$. Equivalently, $Y$ is the union of the strata $X_{\lambda}$ for all partitions $\lambda$, all of whose parts $\lambda_{i}$ are divisible by $l$. We shall prove

Proposition 2.1. $Y$ is a closed $\mu_{q}$-invariant submanifold of $X_{q}$, algebraically isomorphic to the disjoint union of $l$ copies of $X_{r} \times \mathbb{C}^{q-r}$. In terms of the $\mu_{q}$-action

$$
Y \cong \mu_{q} \times \mu_{r}\left(X_{r} \times \mathbb{C}^{q-r}\right) \text {, }
$$

where $\mu_{r}$ acts on $X_{r}$ and on $\mathbb{C}^{q-r}$ by multiplication.

Proposition 2.2.

$$
H^{*}\left(X_{q}-Y\right)_{p}=0 \text {. }
$$

Before proving Propositions 2.1 and 2.2 let us show that they imply Theorem 1.1. The submanifold $Y$ of $X_{q}$ has complex codimension $(2 q-2)-(2 r-2)-(q-$ $r)=q-r$. Let $U$ be a $\mu_{q}$-invariant tubular neighborhood of $Y$ in $X_{q}$. We have

$$
\begin{aligned}
H^{i}\left(X_{q}\right)_{p} & \cong H^{i}\left(X_{q}, X_{q}-Y\right)_{p} \text { from Proposition } 2.2 \\
& \cong H^{i}(U, U-Y)_{p} \text { by excision } \\
& \cong H^{i-2 q+2 r}(Y)_{p} \text { by the Thom isomorphism theorem. }
\end{aligned}
$$

Proposition 2.1 implies that $H^{*}(Y)$ is the representation of $\mu_{q}$ induced from the representation $H^{*}\left(X_{r}\right)$ of $\mu_{r}$. It follows that $H^{i}\left(X_{q}\right)_{p} \cong H^{i-2 q+2 r}\left(X_{r}\right)_{p}$. This completes the proof of Theorem 1.1, for we can assume by induction that the theorem is true when $q$ is replaced by $r$. 
Proof of Proposition 2.1. If $\varphi / \psi=\varphi / \chi^{l}$ belongs to $Y$, then $\mathscr{R}(\varphi, \psi)=\mathscr{R}(\varphi, \chi)^{l}$, so $\mathscr{R}(\varphi, \chi)$ is an $l^{\text {th }}$ root of unity, and $Y$ breaks up into $l$ components, $Y_{u}$, according to the value of $u=\mathscr{R}(\varphi, \chi)$. We can write

$$
\varphi=\varphi_{0} \chi+\varphi_{1}
$$

uniquely, where $\varphi_{1}$ has degree less than $r$, and $\varphi_{0}$ has degree less than $q-r$. Then $\mathscr{R}(\varphi, \chi)=\mathscr{R}\left(\varphi_{1}, \chi\right)$, so the correspondence

$$
\varphi / \chi^{l} \leftrightarrow\left(\varphi_{1} / \chi, \varphi_{0}\right)
$$

is an isomorphism between $Y_{1}$ and $X_{r} \times \mathbb{C}^{q-r}$. Finally, multiplication by $\zeta \in \mu_{q}$ is an isomorphism $Y_{1} \rightarrow Y_{\zeta^{r}}$.

Proof of Proposition 2.2. We begin with

Lemma 2.3. Suppose that $q=q_{1}+q_{2}$, and that $U$ is the open $\mu_{q}$-invariant subset of $X_{q}$ consisting of all rational functions $\varphi / \psi$ with $\psi \in W$, where $W$ is an open subset of the space of monic polynomials such that all $\psi \in W$ can be factorized $\psi=\psi_{1} \psi_{2}$ into ordered coprime factors $\psi_{1}, \psi_{2}$ depending continuously on $\psi \in W$, with $\operatorname{deg}\left(\psi_{i}\right)=q_{i}$. Suppose also that $q_{1}$ and $q_{2}$ are not divisible by the order of $e^{2 \pi i p / q}$. Then $H^{*}(U)_{p}=0$.

Proof of the Lemma. If $\varphi / \psi \in U$ we have a unique decomposition

$$
\frac{\varphi}{\psi}=\frac{\varphi_{1}}{\psi_{1}}+\frac{\varphi_{2}}{\psi_{2}} .
$$

We can define an action of the group

$$
G=\left\{\left(\zeta_{1}, \zeta_{2}\right) \in \mathbb{T} \times \mathbb{T}: \zeta_{1}^{q_{1}} \zeta_{2}^{q_{2}}=1\right\}
$$

on $U$ by

$$
\left(\zeta_{1}, \zeta_{2}\right) \cdot(\varphi / \psi)=\zeta_{1}\left(\varphi_{1} / \psi_{1}\right)+\zeta_{2}\left(\varphi_{2} / \psi_{2}\right)
$$

For

$$
\begin{aligned}
\mathscr{R}(\varphi, \psi) & =\mathscr{R}\left(\varphi_{1} \psi_{2}+\varphi_{2} \psi_{1}, \psi_{1} \psi_{2}\right) \\
& =(-1)^{q_{1} q_{2}} \mathscr{R}\left(\varphi_{1}, \psi_{1}\right) \mathscr{R}\left(\varphi_{2}, \psi_{2}\right) \mathscr{R}\left(\psi_{1}, \psi_{2}\right)^{2},
\end{aligned}
$$

and so the action of $\left(\zeta_{1}, \zeta_{2}\right) \in G$ multiplies $\mathscr{R}(\varphi, \psi)$ by $\zeta_{1}^{q_{1}} \zeta_{2}^{q_{2}}=1$. The action of $G$ extends the action of the cyclic group $\mu_{q}$, for $\mu_{q}$ can be identified with the diagonal subgroup

$$
\left\{\left(\zeta_{1}, \zeta_{2}\right) \in G: \zeta_{1}=\zeta_{2}\right\} \text {. }
$$

Now $G$ is isomorphic to $\mathbb{T} \times \mu_{d}$, where $d$ is the greatest common divisor of $q_{1}$ and $q_{2}$. This means that if $g$ is a generator of $\mu_{q}$ then the action of $g^{d}$ on $U$ is homotopic to the identity, and hence that $H^{*}(U)_{p}=0$ unless the order of $e^{2 \pi i p / q}$ divides $d$.

Returning to the proof of Proposition 2.2, we now see, by using the MayerVietoris sequence and induction on $n$, that $H^{*}(U)_{p}=0$ if $U$ is the union of $n$ open subsets $U_{1}, \ldots, U_{n}$ of $X_{q}$ which each satisfy the hypotheses of Lemma 2.3. (Notice that $U_{n} \cap\left(U_{1} \cup \cdots \cup U_{n-1}\right)$ is a union of $n-1$ sets which satisfy the hypotheses.) This essentially completes the proof of Proposition 2.2, as the open subset $U=X_{q}-Y$ of $X_{q}$ is a union of such sets $U_{i}$. For if $\psi$ is a monic polynomial 
with a root $\beta$ of multiplicity $q_{1}$ not divisible by $l$ then any polynomial near $\psi$ can be factorized canonically as $\psi_{1} \psi_{2}$, where $\psi_{1}$ has $q_{1}$ roots near $\beta$, and $\psi_{2}$ has no roots near $\beta$. This argument leads to a covering of $U$ by a countable number of subsets $\left\{U_{i}\right\}_{i \in S}$ of the desired type. At first sight the Mayer-Vietoris method works only for a finite covering. But there is a well-known device for dealing with this situation. We choose a partition of unity $\left\{\lambda_{i}\right\}$ subordinate to the covering $\left\{U_{i}\right\}$ of $U$. We can assume that at most $N+1$ of the numbers $\lambda_{i}(x)$ are non-zero at any point $x$ of $U$, where $N=\operatorname{dim}(U)$. Then for each finite subset $\sigma$ of $S$ we define

$$
U_{\sigma}^{\prime}=\left\{x \in U: \inf _{i \in \sigma} \lambda_{i}(x)>\sup _{i \notin \sigma} \lambda_{i}(x)\right\} .
$$

The sets $U_{\sigma}^{\prime}$ cover $U$. Finally, for each $k \in\{0,1, \ldots, N\}$ we define $V_{k}$ as the union of the $U_{\sigma}^{\prime}$ when $\sigma$ runs through the subsets of $S$ with $k+1$ elements. The sets $V_{0}, V_{1}, \ldots, V_{N}$ cover $U$, and each $V_{i}$ satisfies the hypotheses of Lemma 2.3, as it is a disjoint union of subsets each contained in one of the $U_{i}$.

Proof of Theorem 1.3. The simplest proof uses the same argument once again, but applies it to the alternative, more "physical", description of $\mathscr{M}_{q}$ as a space of clusters of monopoles in $\mathbb{R}^{3}$. Unfortunately, this description does not seem to have been worked out with sufficient precision for our purpose, so the following proof must be regarded as heuristic rather than complete. We have therefore given a complete proof in the Appendix, using the description of $\mathscr{M}_{q}$ by rational functions. It avoids the questionable use of clusters, but is much more complicated.

The cluster description depends on the fact that a $q$-monopole $\Phi \in \mathscr{M}_{q}$ centered at $x \in \mathbb{R}^{3}$ has an energy distribution in $\mathbb{R}^{3}$, and can sometimes be interpreted as the superposition of $q_{i}$-monopoles $\Phi_{l}$ centered at $x_{i} \in \mathbb{R}^{3}$ for $i=1,2, \ldots, k$, where $\Sigma q_{i}=q, \Sigma q_{i} x_{i}=q x$, and the points $x_{1}, \ldots, x_{k}$ are far apart in $\mathbb{R}^{3}$. In that case the assignment

$$
\Phi \mapsto\left(T_{-x_{1}} \Phi_{1}, \ldots, T_{-x_{k}} \Phi_{k} ; x_{1}, x_{2}, \ldots, x_{k}\right),
$$

where $T_{y}$ is the operation of translating a monopole by $y \in \mathbb{R}^{3}$, extends to a local diffeomorphism

$$
f: U \rightarrow \mathscr{M}_{q_{1}}^{0} \times \mathscr{M}_{q_{2}}^{0} \times \cdots \times \mathscr{M}_{q_{k}}^{0} \times \mathbb{R}^{3 k}
$$

defined in a neighborhood $U$ of $\Phi$ in $\mathscr{M}_{q}$, where $\mathscr{M}_{q_{i}}^{0}$ denotes the space of centered $q_{i}$-monopoles. We can suppose that the image of $U$ is invariant under the action of $\mathbb{T}^{k}$ on $\mathscr{M}_{q_{1}}^{0} \times \cdots \times \mathscr{M}_{q_{k}}^{0}$ which rotates the phases of the monopoles, and $f$ is automatically $\mathbb{T}$-equivariant for the diagonal action of $\mathbb{T}$ which rotates the overall phase. This enables us to extend the $\mathbb{T}$-action on $U$ to a $\mathbb{T}^{k}$-action.

In the new picture the space $X_{q}$ obtained from $\mathscr{M}_{q}^{0}$ by normalizing the overall phase must not be thought of as a subspace of $\mathscr{M}_{q}^{0}$. It is properly defined as the simply-connected covering space of $\mathscr{M}_{q}^{0} / \mathbb{T}$, whose fundamental group is cyclic of order $q$ : we can identify it with the rational functions $\varphi / \psi$ with $\mathscr{R}(\varphi, \psi)=1$ only after choosing a direction in $\mathbb{R}^{3}$. Nevertheless, a $\mathbb{T}$-invariant open subset $U$ of $\mathscr{M}_{q}^{0}$ defines an open subset $U^{\prime}$ of $X_{q}$ which is the restriction of the $q$-fold covering to $U / \mathbb{T}$. Taking $k=2$ in the preceding discussion, the $\mathbb{T} \times \mathbb{T}$ action on $U$ induces an action on $U^{\prime}$ of the group $G$ which was introduced in 2.4 , but which now appears as the group of transformations of $U^{\prime}$ which cover the action of $(\mathbb{T} \times \mathbb{T}) / \mathbb{T}$ on $U / \mathbb{T}$. Just as in the proof of Lemma 2.3, we can conclude that $H^{*}\left(U^{\prime}\right)_{p}=0$ if $p$ is prime to $q$, which is the only relevant case. 
Now let $V$ be the open subset of $X_{q}$ consisting of all monopoles which can be regarded as superpositions of two widely separated configurations. A basic property of $X_{q}$ is that the complement of $V$ in $X_{q}$ is compact. The argument of the proof of Proposition 2.2 shows that $H^{*}(V)_{p}=0$ if $(p, q)=1$, and hence that all elements of $H^{*}\left(X_{q}\right)_{p}$ come from $H^{*}\left(X_{q}, V\right)_{p}$, and so have compact support.

To conclude this section, let us explain more precisely the mild caution we expressed about the preceding argument. If in an open subset $U$ of $\mathscr{M}_{q}$ the monopoles split into clusters of charge $q_{1}$ and $q_{2}$, with $q_{1}+q_{2}=q$, there seems no real problem in defining a $\mathbb{T}^{2}$-action on $U$ which rotates the phases of the clusters independently. What needs to be checked carefully is that if $U^{\prime}$ is another region with a similar splitting $q=q_{1}^{\prime}+q_{2}^{\prime}$, then in $U \cap U^{\prime}$, where the monopoles break up into, say, three clusters, the $\mathbb{T}^{2}$-action coming from $U$ and $U^{\prime}$ form parts of a $\mathbb{T}^{3}$-action on $U \cap U^{\prime}$ which rotates the phases of the three clusters. This was used, implicitly, in the Mayer-Vietoris argument: essentially it is equivalent to the assertion that superposition of widely separated clusters is an associative operation. (The corresponding assertion in the rational function description is obvious, for there superposition is simply addition of the rational functions.)

\section{3. $L^{2}$ Harmonic Forms in the Coprime Case}

If we are prepared to make some further, rather plausible, assumptions about the metric of the space $X_{q}$ we can use the preceding methods to prove Sen's conjecture completely in the case when $p$ and $q$ are coprime.

Assumptions. The space $\mathscr{M}_{q}^{0}$ of centered monopoles can be covered by a finite number of $\mathbb{T}$-invariant open subsets $V_{0}, \ldots, V_{m}$ with the following properties:

(i) The closure of $V_{0}$ is compact.

(ii) If $i \neq 0$ then $V_{i}$ consists of configurations which can be broken into two ordered widely-separated parts with charges $a_{i}$ and $b_{i}$ such that $a_{i}+b_{i}=q$.

(iii) The action of $\mathbb{T}$ on $V_{i}$ extends to an action of the group $\mathbb{T}^{2}$ which rotates the phases of the parts independently. This action is by near-isometries, in the sense that if $f \in \mathbb{T}^{2}$ then

$$
A^{-1} g \leqq f^{*} g \leqq A g
$$

for some $A \geqq 1$, where $g$ is the metric of $V_{i}$. Furthermore, the orbits of the action of $\mathbb{T}^{2}$ on $V_{i}$ are of bounded size.

(iv) There is a smooth partition of unity $\left\{\lambda_{i}\right\}$ subordinate to the covering $\left\{V_{i}\right\}$ of $\mathscr{M}_{q}^{0}$ such that each $d \lambda_{i}$ is bounded.

The assumption (iv) follows from a stronger statement, with three parts.

(iv) (a) We can take $V_{0}$ to be the interior of a compact smooth manifold $\bar{V}_{0}$ with boundary $Y$, and $\mathscr{M}_{q}^{0}-V_{0}$ is diffeomorphic to $Y \times[0, \infty)$.

(iv) (b) The projection $\pi: \mathscr{M}_{q}^{0}-V_{0} \rightarrow Y$ is length-decreasing for the Riemannian metric.

(iv)(c) The sets $V_{i}$ for $i \neq 0$ are of the form $\pi^{-1}\left(W_{i}\right)$ for some $W_{i} \subset Y$.

We hope to return to the justification of these assumptions in another paper. Meanwhile, let us show that they imply Sen's conjecture when $p$ and $q$ are coprime. 
It is enough to show that

$$
H_{c p t}^{*}\left(X_{q}\right)_{p} \rightarrow \mathscr{H}_{q, p}
$$

is surjective.

For any Riemannian manifold $M$, let $h^{*}(M)$ denote the cohomology of the subcomplex of the de Rham complex of $M$ consisting of smooth forms $\alpha$ such that both $\alpha$ and $d \alpha$ belong to $L^{2}$. If $\mu_{q}$ acts on $M$ by near-isometries, we can calculate the component $h^{*}(M)_{p}$ from the subcomplex of forms $\alpha$ such that $g_{\zeta}^{*} \alpha=\zeta^{p} \alpha$.

For the complete manifold $X_{q}$ it follows from the discussion at the end of Sect. 1 that there is an injective map

$$
\mathscr{H}_{q, p} \rightarrow h^{*}\left(X_{q}\right)_{p}
$$

so to prove the conjecture it is enough to show that $h^{*}\left(V_{1}^{\prime} \cup \cdots \cup V_{m}^{\prime}\right)_{p}=0$, where $V_{i}^{\prime}$ is the $q$-fold covering space of $V_{i} / \mathrm{T}$ described in Sect. 2.

If $M=M_{1} \cup M_{2}$, where $M_{1}$ and $M_{2}$ are open submanifolds of $M$, there is a Mayer-Vietoris sequence

$$
\cdots \rightarrow h^{*}(M) \rightarrow h^{*}\left(M_{1}\right) \oplus h^{*}\left(M_{2}\right) \rightarrow h^{*}\left(M_{1} \cap M_{2}\right) \rightarrow \cdots,
$$

providing there is a partition of unity $\lambda_{1}+\lambda_{2}=1$ for $M=M_{1} \cup M_{2}$ for which $d \lambda_{1}=-d \lambda_{2}$ is bounded. There is also a generalised Mayer-Vietoris argument applying to coverings by more than two sets which implies that if there is a partition of unity, $\left\{\lambda_{i}\right\}$, for $M=M_{1} \cup \cdots \cup M_{m}$, with each $d \lambda_{i}$ bounded and if $h^{*}\left(\bigcap_{i \in A} M_{i}\right)=0$ for all nonempty $A \subseteq\{1, \ldots, m\}$, then $h^{*}(M)=0$ (this follows by considering the double complex ). Consequently we have that $h^{*}\left(V_{1}^{\prime} \cup \cdots \cup V_{m}^{\prime}\right)_{p}=0$, in view of the following variant of Lemma 2.3, where $G_{i}$ denotes the group defined by (2.4), but with $\left(q_{1}, q_{2}\right)$ replaced by $\left(a_{i}, b_{i}\right)$.

Lemma 3.1. If $M$ is a $G_{i}$-stable open submanifold of $V_{i}^{\prime}$, and $p$ and $q$ are coprime, then $h^{*}(M)_{p}=0$.

Proof. Let $\alpha$ be a smooth $L^{2}$ form representing an element of $h^{*}(M)_{p}$. We have $g_{\zeta}^{*} \alpha=\zeta^{p} \alpha$, where $\zeta$ is a primitive $q^{\text {th }}$ root of unity. The element $\varphi=g_{\zeta}^{d}$, where $d=G C D\left(a_{l}, b_{i}\right)$, belongs to the identity component of $G_{i}$, so we have an explicit homotopy $\varphi \simeq$ (identity) generated by a bounded vector field. This shows that $\varphi^{*} \alpha-\alpha=d \beta$ for some $L^{2}$ form $\beta$. But $\varphi^{*} \alpha=\zeta^{p d} \alpha$, and $\zeta^{p d} \neq 1$. So $\alpha=d\left(\zeta^{p d}-\right.$ $1)^{-1} \beta$ ), as we want.

\section{Appendix}

Here we calculate the map $H_{c p t}^{2 q-2}\left(X_{q}\right) \rightarrow H^{2 q-2}\left(X_{q}\right)$. In what follows, $p$ and $q$ are coprime, $\zeta=e^{2 \pi i / q}$, and $g$ is the generator of $\mu_{q}$ corresponding to multiplication of rational functions by $\zeta$. It will be convenient to work in homology - there are natural isomorphisms:

$$
H_{2 q-2}\left(X_{q}\right)_{p}=H_{c p t}^{2 q-2}\left(X_{q}\right)_{p}=\left(H^{2 q-2}\left(X_{q}\right)_{-p}\right)^{*}=\left(H_{2 q-2}^{c}\left(X_{q}\right)_{-p}\right)^{*},
$$

where the last of these spaces, $H^{c}$, the closed chain homology, is introduced only to define a normalisation for Theorem A.1. In this language there is an intersection 
pairing between $H_{2 q-2}\left(X_{q}\right)$ and $H_{2 q-2}^{c}\left(X_{q}\right)$, which is perfect by Poincaré duality, and another between $\mathrm{H}_{2 q-2}\left(X_{q}\right)$ and itself, which we should like to prove is perfect.

We observe that the argument of Proposition 2.1 with $l=q$ implies that

$$
\tau_{p}=\frac{1}{q} \sum_{k<q} \zeta^{-k p} Y_{\zeta^{k}}=\frac{1}{q} \sum_{k<q} \zeta^{-k p} g^{k} Y_{1}
$$

is a generator of $H_{2 q-2}^{c}\left(X_{q}\right)_{p}$. (The submanifold $Y_{\zeta^{k}}$ being complex has a standard orientation.)

Theorem A.1. If $\sigma_{p} \in H_{2 q-2}\left(X_{q}\right)_{p}$ is the dual basis to $\tau_{-p}$ (i.e., $\left.\sigma_{p} \tau_{p^{\prime}}=\delta_{p,-p^{\prime}}\right)$, then $\sigma_{p} \sigma_{p^{\prime}}=(-1)^{q-1} \delta_{p,-p^{\prime}}$.

Corollary. The map $H_{c p t}^{2 q-2}\left(X_{q}\right) \rightarrow H^{2 q-2}\left(X_{q}\right)$ is an isomorphism.

Remark. Transforming $\left\{\sigma_{p}\right\}$ to a real basis we can calculate the signature of the pairing, with the result that it is positive definite if $q$ is odd and negative definite if $q$ is even.

Proof of Theorem A.1. A typical rational function in $X_{q}$ is denoted by $\varphi(z) / \psi(z)$, the roots of $\psi$ being $\left\{z_{1}, \ldots, z_{q}\right\}$. Recall that $\sum z_{i}=0$ and $\prod \varphi\left(z_{i}\right)=1$. Let $\varepsilon$ be a fixed positive number, taken sufficiently small in what follows.

Define the (closed) subset $F \subseteq \mathbb{C}^{q} \times \mathbb{T}^{q}$ by

$$
F=\left\{\left(z_{1}, \ldots, z_{q}, w_{1}, \ldots, w_{q}\right): \sum z_{i}=0, \prod w_{i}=1, w_{i} \neq w_{j} \Rightarrow\left|z_{i}-z_{j}\right| \geqq \varepsilon\right\} .
$$

Define the map $\beta: F \rightarrow X_{q}$ by prescribing $\varphi\left(z_{i}\right)=w_{i}$ and $\psi\left(z_{i}\right)=0$ if $z_{i}$ are distinct (i.e., the map of Lagrange interpolation). This extends smoothly to the whole of $F$ because of the $w_{i} \neq w_{j} \Rightarrow\left|z_{i}-z_{j}\right| \geqq \varepsilon$ condition.

The objective will be to describe explicitly the generating cycles of $H_{2 q-2}\left(X_{q}\right)$ and then calculate their intersections by using a convenient diffeomorphism of $X_{q}$ to perturb $\sigma_{p}$. The idea is that the proof of Theorem 1.1 implies that $\beta_{*}: H_{2 q-2}(F) \rightarrow$ $H_{2 q-2}\left(X_{q}\right)$ is surjective, so that we may work mainly in $F$. In fact, rather than describing the complete cycles, it will prove simpler to use relative cycles (see below).

Recall that the proof of Lemma 2.3 made use of homotopies which were only defined when the roots of $\psi$ were separated into two sets. This means that it is necessary to split $X_{q}$ into subsets in which the $z_{i}$ are separated in a controlled manner. To do this we introduce the permutahedron, $P_{q}$ in $\mathbb{R}^{q}$.

Choose $q$ distinct real numbers $r_{1}<r_{2}<\cdots<r_{q}$ such that $\sum r_{i}=0$. (The $r_{i}$ will have to be chosen sufficiently small to make a later part of this argument work.) Define $P\left(a_{1}, \ldots, a_{n}\right)$ to be the convex hull in $\mathbb{R}^{n}$ of the $n$ ! points $\left(a_{\pi_{1}}, \ldots, a_{\pi_{n}}\right)$, where $\pi \in S_{n}$ is a permutation. Let $P_{q}=P\left(r_{1}, \ldots, r_{q}\right)$, a polyhedron of dimension $q-1$. If $A=\left\{a_{1}, \ldots, a_{k}\right\} \subseteq\{1,2, \ldots, q\}$ with $a_{1}<\cdots<a_{k}$, then define $\pi_{A}: \mathbb{R}^{q} \rightarrow \mathbb{R}^{k}$ by $\pi_{A}\left(x_{1}, \ldots, x_{q}\right)=\left(x_{a_{1}}, \ldots, x_{a_{k}}\right)$. If $A_{1}, \ldots, A_{r}$ is an ordered partition of $\{1,2, \ldots, q\}$ into non-empty subsets then let $s_{i}=\sum_{j<i}\left|A_{j}\right|$ and define

$$
\left(A_{1}|\cdots| A_{r}\right)=\left\{x \in \mathbb{R}^{q}: \pi_{A_{i}}(x) \in P\left(r_{s_{l}+1}, \ldots, r_{s_{i+1}}\right)\right\} .
$$

It is a fact that $\left(A_{1}|\cdots| A_{r}\right)$ is a face of $P_{q}$ and that all faces arise in this way. So faces of $P_{q}$ correspond to ordered partitions and are isomorphic to products of lower dimensional permutahedra. 
The property of these faces we shall need is that if $\left(x_{1}, \ldots, x_{q}\right) \in\left(A_{1}|\cdots| A_{r}\right)$ and $i \neq j$ then $\left|x_{a}-x_{b}\right| \geqq \varepsilon$ whenever $a \in A_{i}$ and $b \in A_{j}$. This is ensured by choosing $\varepsilon$ smaller than $r_{2}-r_{1}, r_{3}-r_{2} \ldots, r_{q}-r_{q-1}$.

We wish to think of the faces of $P_{q}$ as singular chains, so we need to give them an orientation. This will be done by giving a section of the top exterior power of the tangent bundle. If $A \subseteq\{1, \ldots, q\}$ then define

$$
O_{A}=\left(\frac{\partial}{\partial x_{a_{2}}}-\frac{\partial}{\partial x_{a_{1}}}\right) \wedge \cdots \wedge\left(\frac{\partial}{\partial x_{a_{k}}}-\frac{\partial}{\partial x_{a_{1}}}\right),
$$

where $A=\left\{a_{1}, \ldots, a_{k}\right\}$ and $a_{1}<\cdots<a_{k}$. It is convenient to define the orientation of $\left(A_{1}|\cdots| A_{r}\right)$ to be

$$
\operatorname{sgn}\left(A_{1} A_{2} \cdots A_{r}\right) \prod_{i=1}^{r}\left[(-1)^{(r-i)\left|A_{i}\right|} O_{A_{i}}\right],
$$

where $\operatorname{sgn}\left(A_{1} A_{2} \cdots A_{r}\right)$ denotes the sign of the permutation in $S_{q}$ obtained by listing the elements of $A_{1}$ in ascending order followed by those of $A_{2}$, and so on. This strange choice makes the formula for the boundary simple:

$$
\partial\left(A_{1}|\cdots| A_{r}\right)=\sum_{i=1}^{r}(-1)^{i} \sum_{\emptyset \neq B \subsetneq A_{l}}\left(A_{1}|\cdots| A_{i-1}|B| A_{i} \backslash B\left|A_{i+1}\right| \cdots \mid A_{r}\right) .
$$

We shall also need to define the homotopy as used in Lemma 2.3. If $R \subseteq$ $\{1, \ldots, q\}$, let $n_{i}=q-|R|$ if $i \in R$ and $n_{i}=-|R|$ otherwise. Then

$$
T_{R}\left(\theta, z_{1}, \ldots, z_{q}, w_{1}, \ldots, w_{q}\right)=\left(z_{1}, \ldots, z_{q}, w_{1} e^{i n_{1} \theta}, \ldots, w_{q} e^{i n_{q} \theta}\right)
$$

is a partially defined map from $[0,2 \pi / q] \times F$ to $F$. If $\sigma$ is a $r$-chain in $F$, such that $T_{R}$ is everywhere defined on $[0,2 \pi / q] \times|\sigma|(|\sigma|$ being the carrier of $\sigma)$, then $T_{R}(\sigma)$ defines in an obvious way an $r+1$-chain whose orientation we shall take to be $O_{T_{R}(\sigma)}=\frac{\partial}{\partial \theta} \wedge O_{\sigma}$. The basic property of $T_{R}$ is

$$
\partial T_{R}+T_{R} \partial=g^{-|R|}-1 .
$$

Now, there is a map

defined by

$$
P_{q} \times P_{q} \times \mathbb{Z} / q \mathbb{Z} \stackrel{\alpha}{\rightarrow} F
$$

$$
\alpha\left(x_{1}, \ldots, x_{q}, y_{1}, \ldots, y_{q}, k\right)=\left(x_{1}+i y_{1}, \ldots, x_{q}+i y_{q}, \zeta^{k}, \ldots, \zeta^{k}\right) .
$$

The desired cycle in $F$ will be built up from linear combinations of the chains

$$
T_{R_{1}} \cdots T_{R_{r-1}} \alpha\left(\left(A_{1}|\cdots| A_{r}\right),(12 \cdots q), k\right) .
$$

It follows from the above definitions that the above chain is defined when each $R_{i}$ is a union of a subcollection of the sets $A_{1}, \ldots, A_{r}$. This is because being in the face $\left(A_{1}|\cdots| A_{r}\right)$ guarantees us that if $a \in A_{i}, b \in A_{j}, i \neq j$ then $x_{a}$ and $x_{b}$, hence $x_{a}+i y_{a}$ and $x_{b}+i y_{b}$, are separated, which allows us to move $w_{a}$ apart from $w_{b}$.

Note that we only use the top dimensional face of $P_{q}$ to restrict the imaginary parts of the $z_{i}$. This will result in a cycle relative to the subset $F^{0}$ of $F$ defined by

$$
F^{0}=\left\{\left(z_{1}, \ldots, z_{q}, w_{1}, \ldots, w_{q}\right):\left(\operatorname{Im}\left(z_{1}\right), \ldots, \operatorname{Im}\left(z_{q}\right)\right) \in \partial P_{q}\right\}
$$


and on applying $\beta$, a cycle relative to the subset $X^{0}$ of $X_{q}$ defined by

$$
X^{0}=\left\{\varphi / \psi:\left|\varphi\left(z_{i}\right)\right|=1,\left(\operatorname{Im}\left(z_{1}\right), \ldots, \operatorname{Im}\left(z_{q}\right)\right) \in \partial P_{q}\right\} .
$$

(This definition makes sense because $P_{q}$ is invariant under permutation of coordinates.)

Define

$$
\left[A_{1}|\cdots| A_{r}\right]_{p}=\sum_{k<q} \zeta^{-p k} \alpha\left(\left(A_{1}|\cdots| A_{r}\right),(12 \cdots q), k\right),
$$

so $\left[A_{1}|\cdots| A_{r}\right]_{p}$ is a " $p$-type" chain, that is $g\left[A_{1}|\cdots| A_{r}\right]_{p}=\zeta^{p}\left[A_{1}|\cdots| A_{r}\right]_{p}$. Let $U_{R}=\left(\zeta^{-p|R|}-1\right)^{-1} T_{R}$, so that

$$
\partial U_{R}+U_{R} \partial=1
$$

on $p$-type chains. Here it is used that $p$ and $q$ are coprime.

We are now in a position to construct the cycle we are interested in. Let

$$
\sigma_{p}=\beta\left[\frac{1}{q !} \sum_{\left(A_{l}\right)}(-1)^{\left(\begin{array}{r}
r-1 \\
2
\end{array}\right)} U_{A_{1}} U_{A_{1} \cup A_{2}} \cdots U_{A_{1} \cup \cdots \cup A_{r-1}}\left[A_{1}|\cdots| A_{r}\right]_{p}\right],
$$

the sum being over all ordered partitions of $\{1, \ldots, q\}$. Note that $g T_{R}=T_{R} g$, so all terms are of $p$-type. It follows from (A.2) that, modulo chains in $F^{0}$,

$$
\partial\left[A_{1}|\cdots| A_{r}\right]_{p}=\sum_{i=1}^{r}(-1)^{i} \sum_{\emptyset \neq B \subsetneq A_{l}}\left[A_{1}|\cdots| A_{i-1}|B| A_{i} \backslash B\left|A_{i+1}\right| \cdots \mid A_{r}\right]_{p} .
$$

Some checking using the relation (A.3) then shows that $\sigma_{p}$ is a relative cycle in the pair $\left(X_{q}, X^{0}\right)$.

To calculate the intersection number $\sigma_{p} \sigma_{p^{\prime}}$ we proceed as follows. A map $m: X_{q} \rightarrow X_{q}$ is exhibited such that the following properties hold:

(i) $m$ is a diffeomorphism.

(ii) $m\left(X^{0}\right) \cap \beta(F)=m\left(X^{0}\right) \cap X^{0}=\emptyset$.

(iii) Suppose $\lambda_{1} c_{1}$ and $\lambda_{2} c_{2}$ are component chains in $m\left(\sigma_{p}\right)$ and $\sigma_{p^{\prime}}$ respectively. That is, $\lambda_{i} \in \mathbb{C}$ and, for $i=1,2, c_{i}$ is a map from some polyhedron, $S_{i}$, in $\mathbb{R}^{2 q-2}$ together with an orientation of $S_{i}$. Suppose also that $c_{1}\left(x_{1}\right)=c_{2}\left(x_{2}\right)=y$. Then $x_{i} \notin \partial S_{i}, c_{i}$ is smooth in a neighbourhood of $x_{i}$ and $c_{1} \cap c_{2}$ is transverse at $x_{1}, x_{2}\left(c_{1 *} T_{x_{1}} S_{1}+c_{2 *} T_{x_{2}} S_{2}=T_{y} X_{q}\right)$.

It follows from these conditions that we can calculate $\sigma_{p} \sigma_{p^{\prime}}$ by summing $\lambda_{1} \lambda_{2}$ over pairs $\left(x_{1}, x_{2}\right)$ as in (iii) with the usual sign. To see this we use the general theory of [de R], Sect. 20 together with the following observation. The proof of Lemma 2.3 implies that $H_{2 q-2}\left(X^{0}\right)_{p}=0$. Therefore there is a cycle $\sigma_{p}+\alpha_{p}$ of $X_{q}$ with $\left|\alpha_{p}\right| \subseteq X^{0}$. Condition (ii) above now assures us that $\alpha_{p}$ does not enter into the intersection calculation.

Exactly similar reasoning allows us to calculate the intersection numbers $\sigma_{p} \tau_{p^{\prime}}$ by moving $\tau_{p^{\prime}}$. This is easier, so let us deal with it first. Move $Y_{\omega}$ from $\left\{\varphi / z^{q}\right.$ : $\varphi(0)=\omega\}$ to $Y_{\omega}^{\prime}=\{\varphi / \psi:|\varphi(0)-\omega|<\delta\}$ for some small $\delta>0$ and some fixed choice of $\psi(z)$ whose roots are small and distinct. It is clear that the only term in (A.4) which intersects this is the "top" term, i.e., the one with $r=1$. Taking 
the orientations into consideration we have $\sigma_{p} \tau_{p^{\prime}}=\beta\left(\frac{1}{q !}[12 \cdots q]_{p}\right) \frac{1}{q} \sum_{s} \zeta^{-p^{\prime} s} Y_{\zeta^{s}}^{\prime}=$ $S \frac{1}{q \cdot q !} \sum_{r, s} \zeta^{-p r+p^{\prime} s} \delta_{r s} q !=S \delta_{p,-p^{\prime}}$, where $S= \pm 1$ is a overall sign (depending only on $q$ ) which is unimportant since Theorem A.1 is asserting something about $\sigma_{p} \sigma_{p^{\prime}}$.

Returning to $\sigma_{p} \sigma_{p^{\prime}}$, define $m$ as the composition of two maps, $m_{1}$ and $m_{2}$.

(i) The map $m_{1}$ takes $\varphi / \psi$ to

$$
\frac{\varphi(z) e^{i z} \bmod \psi(z)}{\psi(z)} .
$$

(Here $\varphi(z) e^{i z} \bmod \psi(z)$ means the unique polynomial of degree less than $q$ congruent to $\varphi(z) e^{i z}$ modulo $\psi(z)$.)

(ii) To define the map $m_{2}$ we fix a real number $\lambda$ with $0<\lambda<1$, and map $\varphi / \psi$ to

$$
\frac{\varphi(z / \lambda)}{\lambda^{q} \psi(z / \lambda)}
$$

Both maps are analytic diffeomorphisms (since $\psi$ is always monic) which preserve the resultant, so we may define $m$ to be $m_{2} \circ m_{1}$.

If $m(\varphi / \psi)=\varphi_{m} / \psi_{m}$, where the roots of $\psi_{m}$ are $z_{i}^{\prime}$ and $\varphi_{m}\left(z_{i}^{\prime}\right)=w_{i}^{\prime}$, then

$$
w_{i}^{\prime}=e^{i z_{l}} w_{i} \quad \text { and } \quad z_{i}^{\prime}=\lambda z_{i} .
$$

This establishes property (ii) of $m$, since the condition $\left|w_{i}^{\prime}\right|=1$ implies that $\operatorname{Im}\left(z_{i}\right)=0$. Furthermore, since $\lambda P_{q} \cap \partial P_{q}=\emptyset$, we are reduced to finding intersections of $m\left(\sigma_{p}\right)$ with the top term, $\beta\left(\frac{1}{q !}[12 \cdots q]_{p^{\prime}}\right)$, of $\sigma_{p^{\prime}}$. This implies that $\varphi_{m}$ is a constant polynomial.

Consider now the term in (A.4) corresponding to the partition $A_{1}, \ldots, A_{r}$, and suppose that $\varphi / \psi$ is a point of $\left|\sigma_{p}\right|$ such that $m(\varphi / \psi)=\varphi_{m} / \psi_{m}$ is in $\left|\sigma_{p^{\prime}}\right|$. We have that $w_{1}^{\prime}=w_{2}^{\prime}=\cdots=\zeta^{s}$ for some $s$. If $a, b \in A_{i}$ then $w_{a}=w_{b}$, so $e^{i z_{a}}=e^{i z_{b}}$, and since the $r_{i}$ were chosen sufficiently small, we have $z_{a}=z_{b}$. (This means that intersections can only occur at points arising from the barycentres of faces of $P_{q}$.) There is one more restriction on the intersection points we need to determine, namely that $r=q$, in other words $\left|A_{i}\right|=1$ for all $i$. To see this, let $v_{i}=z_{a_{i}}$ for some (all) $a_{i} \in A_{i}$, and let $\psi_{i}(z)=\left(z-v_{i}\right)^{\left|A_{i}\right|}$. Decompose $\zeta^{s} / \psi$ into partial fractions:

$$
\frac{\zeta^{s}}{\psi}=\sum_{i=1}^{r} \frac{\varphi_{i}}{\psi_{i}}
$$

Then it follows from (A.5) and the nature of the map $\beta$ that

$$
\frac{\varphi}{\psi}=\sum_{i=1}^{r} \frac{e^{-i v_{i}} \varphi_{i}}{\psi_{i}},
$$

and so

$$
m_{1}\left(\frac{\varphi}{\psi}\right)=\sum_{i=1}^{r} \frac{e^{i\left(z-v_{l}\right)} \varphi_{i} \bmod \psi_{i}}{\psi_{i}} .
$$

But this must have constant numerator when put over the common denominator, so it is a constant multiple of $\sum \varphi_{i} / \psi_{i}$. Consequently $e^{i\left(z-v_{i}\right)} \varphi_{i} \equiv C \varphi_{i} \bmod \psi_{i}$. Multiplying by $\prod_{j \neq i} \psi_{j}$ tells us that $e^{i\left(z-v_{i}\right)} \equiv C \bmod \left(z-v_{i}\right)^{\left|A_{i}\right|}$ which is impossible unless $\left|A_{i}\right|=1$. 
This completes the identification of the intersection points $m\left(\left|\sigma_{p}\right|\right) \cap\left|\sigma_{p^{\prime}}\right|$ which, to summarize, arise from the $r=q$ terms in (A.4) with $w_{i}=\zeta^{s} e^{-i z_{i}}$ for some $s$. At such points of $\left|\sigma_{p}\right|$, the $z_{i}$ are all distinct (being equal to a permutation of $r_{1}, \ldots, r_{q}$ ), and the $w_{i}$ are not equal to $q^{\text {th }}$ roots of unity. This shows that property (iii) of $m$ holds, with the possible exception of the transversality condition.

We now have to work out the intersection of

$$
m \beta\left(U_{B_{1}} U_{B_{2}} \cdots U_{B_{q-1}} \alpha\left(\left(a_{1}|\cdots| a_{q}\right),(12 \cdots q), r\right)\right)
$$

with

$$
\beta \alpha((12 \cdots q),(12 \cdots q), s)
$$

where $\left(a_{i}\right)$ is a permutation of $1,2, \ldots, q$ and $B_{i}=\left\{a_{1}, \ldots, a_{i}\right\}$. This amounts to counting solutions of the equations

$$
\sum_{j=1}^{q-1}\left[\left(q I_{i \leqq j}-j\right) \theta_{j}\right]+r_{i} \equiv \frac{2 \pi}{q}(s-r) \bmod 2 \pi,
$$

where $i$ ranges from 1 to $q$ (one of these equations being redundant), $0 \leqq \theta_{j} \leqq 2 \pi / q$ and $I_{i \leqq j}=1$ if $i \leqq j$ and $=0$ otherwise. These equations have a solution only when $s=r$, namely $\theta_{j}=\left(r_{j+1}-r_{j}\right) / q$.

It remains to calculate the sign associated to this intersection (and in doing so check that it is transverse). We omit the linear algebra, but the outcome is that the intersection number from (A.6) is $\delta_{r s}(-1)^{\left(\begin{array}{c}q \\ 2\end{array}\right)} q !^{2} / q$ (making use of the fact that $\left.\prod_{r=1}^{q-1}\left(1-\zeta^{-p r}\right)=q\right)$. This means that

$$
\begin{aligned}
\sigma_{p} \sigma_{p^{\prime}} & =\sum_{r, s<q} \frac{1}{q !} \zeta^{-p r}(-1)^{\left(\begin{array}{c}
q-1 \\
2
\end{array}\right)} \zeta^{-p^{\prime} s} \frac{1}{q !} \delta_{r s}(-1)^{\left(\begin{array}{l}
q \\
2
\end{array}\right)} q !^{2} / q \\
& =(-1)^{q-1} \delta_{p,-p^{\prime}} .
\end{aligned}
$$

\section{References}

[A-H] Atiyah, M.F., Hitchin, N.J.: The geometry and dynamics of magnetic monopoles. Oxford: Oxford Univ. Press, 1988

[de R] de Rham, G.: Differential manifolds. English edition. Berlin, Heidelberg, New York: Springer, 1984

[S] Segal, G.B.: The topology of spaces of rational functions. Acta Mathematica 143, 39-72 (1979)

[Sen] Sen, A.: To appear (HEP preprints: hep-th/9402002 and hep-th/9402032)

Communicated by R.H. Dijkgraaf 
